\title{
Unsteady Viscous Flow Simulation around the Blade of Rowing Boat
}

\author{
by Todor A. Videv*, Member Yasuaki Doi*, Member
}

\begin{abstract}
Summary
Some aspects of unsteady viscous flow field around an oar blade of a rowing boat are studied numerically by means of laminar, incompressible unsteady full 2D Navier-Stokes simulation. Main objectives are to reveal the flow phenomena in order to understand the mechanism of generation of the propulsive force and to study the influence of different parameters on the flow pattern and efficiency of rowing. The main parameters under consideration are: 1) angular velocity of the blade (speed of rowing), 2) blade geometry and 3) outboard length of the oar. Special attention is payed to the unsteady effects taking place during the blade stroke. An analysis of the accuracy and reliability of the results with respect to the limited Reynolds number and $2 \mathrm{D}$ simulation is provided.

Analysis of the results for the flow simulation and hydrodynamical forces acting on the blade during its stroke shows, that the flow is very sensitive even to the small changes of main motion or geometry parameters, although, a general flow pattern is valid for all of the studied cases.

Performed flow simulation shows that for all studied cases, strong and stable vortical structures, formed near the catch position, attend the blade motion and have strong influence on the further flow evolution. This fact determines the strong unsteady effects on the flow pattern and the exerted hydrodynamical forces.
\end{abstract}

\section{Introduction}

For centuries, shells, oars and blades of rowing boats have been evaluated in order to have at present time much lighter, much more durable and from many points of view-more efficient rowing equipment. Excluding oarsman abilities and skills, such drastic changes may be explained by high technologies and up-to-date materials, as well as with a better understanding of the physical aspects of the flow around a blade in order to improve its efficiency during a rowing stroke.

The hydrodynamical aspects of shells were studied by Ikehata et al. ${ }^{1)}$. Investigations on oar motion and performance, which mainly cover the mechanical aspects of the complicated dynamic system of shell, oarsmen and rowing oars, were studied by Hayashi et al. ${ }^{2)}$. Recently, an original method combining image processed fullscale data, numerical modelling and experimental results, developed by Amo et al. ${ }^{3}$ was reported by Mori et al. ${ }^{4}$. Complete investigations followed by interesting discussion were presented by Suzuki ${ }^{5}$. These studies present a progress in understanding of rowing, however, the hydrodynamical forces acting on a blade are obtained assuming a pseudo-steady flow around a blade.

\footnotetext{
* Faculty of Engineering, Hiroshima University
}

Received 11th Jan 1993

Read at the Spring meeting 19, 20th May 1993
Present study concerns unsteady hydrodynamical aspects of a rowing oar blade with assumption that the flow is laminar $\left(R e=1.0 \times 10^{3}\right)$, two-dimensional (without free surface) and constant boat speed. The affect of the oar on the flow is neglected. Main objectives are to reveal the common flow features and the unsteady effects taking place during the blade pitching. To investigate the flow and forces around the blade, the dependency on some major parameters as blade geometry, blade angular velocity and outboard oar length is studied.

The numerical unsteady flow simulation is based on a 2D full Navier-Stokes Eqs. solver, in vorticity-stream function formulation, assuming laminar viscous flow around the blade. This method was successfully used for the investigation of the unsteady flow around the pitching 2D hydrofoil ${ }^{6), 7)}$.

A brief explanation of the formulation, used numerical algorithm, and blade motion model are presented with remarks on the numerical accuracy. At first, a general discussion of the main features of the unsteady flow is presented. This discussion is followed by analysis of the influence of some primary parameters as angular velocity of the oar blade, the blade geometry and outboard oar length (pivot point location). Presented discussion is closed by an efficiency analysis and more general conclusions. 


\section{Blade Motion Model}

The boat motion is assumed constant. With such assumption, the problem may be defined as a stroke motion of an oar in a uniform free stream with velocity $U_{\infty}$, equal to the speed of the boat with opposite sign. Fig. 1 illustrates the shell of the boat with an oar at a fixed angular position. Used global inertial coordinate system fixed to the boat and noninertial rotating coordinate system fixed to the tip of the blade are presented.

The stages of the blade entry at a catch angle and a finish can not be modelled by the present method. According to the limitations of the used formulation, the blade motion is divided into two parts. The preliminary stage consists of impulsive start at zero incidence until the flow is fully developed. The second stage is a blade pitching in stroke from $\beta=0$ [deg] to $\beta=135$ [deg] (see Fig. 1) when the finish is passed. The main parameters of the blade motion are the outboard oar length (distance to the pivot point location) $r_{p}$ (Fig. 1), frequency of pitching $f$, mean angular position $\beta_{M}$ (position of maximum angular velocity), and amplitude of pitching $\beta_{A}$. The dependence of the oar angular position on these parameters defines the pitching mechanism during the stroke $\beta(t)=\beta\left(r, f, \beta_{M}, \beta_{A}, t\right)$. It is convenient to use the normalized values of the main parameters introducing normalized time $t^{*}=t U_{\infty} / c(c$, chord length of the blade), normalized pivot poiot location $r_{p}^{*}$ $=r_{p} / c$, and reduced frequency $k=f_{C} / 2 U_{\infty}$. The reference outboard oar length $l_{2}$ is defined as the distance from the pivot point (oar collar) to the middle of the blade (Fig. 1). This length is connected with the parameter $r_{p}$ by the relation $l_{2}=r_{p}-c / 2$ or $l_{2}^{*}=r_{p}^{*}-0.5$.

The oar motion is modelled as sinusoidal. In practice, it will depend on the oarsman style of rowing and many other parameters.

It is assumed, that after the developed flow is obtained at $\beta=0[\mathrm{deg}]$, the blade is pitching according to a cosine law with parameters $\beta_{M}=\beta_{A}=90[\mathrm{deg}]$. If the time when the pitching started $t_{p}$ is taken as a reference time, the stages of blade motion can be described as:

$$
\begin{aligned}
& \beta(t)=0.0, \quad\left(0 \leq 0 \equiv t_{p}\right), \\
& \beta(t)=\beta_{M}-\beta_{A} \cos (f t), \quad\left(t>t_{p}\right) .
\end{aligned}
$$

The motion with such parameters prescribes zero angular velocity at $\beta=0[\mathrm{deg}]$, a finite angular velocity at a catch angle (about $45[\mathrm{deg}]$ ), a maximum angular velocity at $90[\mathrm{deg}]\left(\beta=\beta_{M}\right)$ and a finite angular velocity at the finish (about $120[\mathrm{deg}]$ ). The paths of the blade, during the stroke, are illustrated in Fig. 2.

The angular velocity and the acceleration of the blade $\Omega=d \beta / d t$ and $\theta=d^{2} \beta / d t^{2}$ are :

$$
\begin{aligned}
& \Omega(t)=\frac{2 U_{\infty} k}{c} \beta_{A} \sin \left(2 k t^{*}\right), \\
& \vartheta(t)=\frac{\left(2 U_{\infty} k\right)^{2}}{c^{2}} \beta_{A} \cos \left(2 k t^{*}\right) .
\end{aligned}
$$

The velocity of the blade with respect to its middle point $v$ (see Fig. 1) is $v=l_{2} \Omega$. Blade rotation $\Omega(t)$ in the

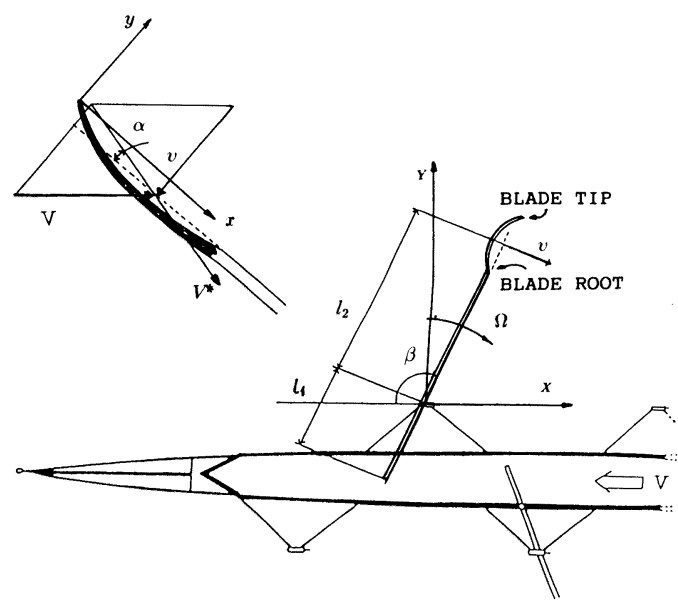

Fig. 1 Coordinate Systems and Definitions.

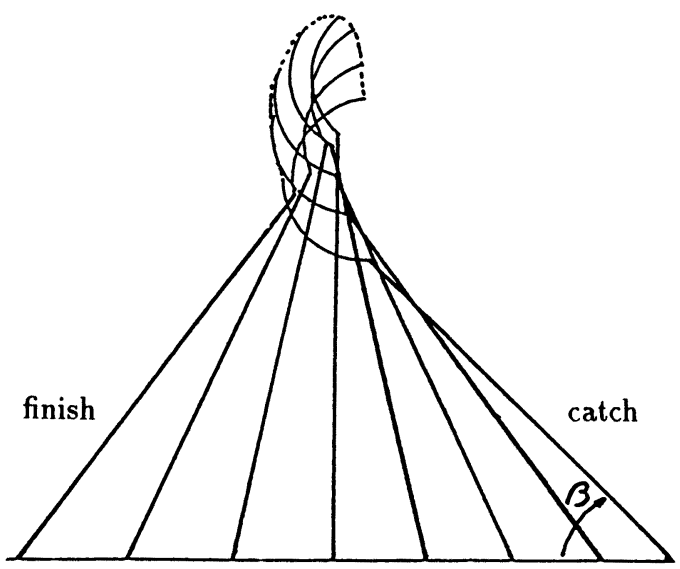

Fig. 2 Paths of Oar and Blade per a Stroke.

uniform free stream $U_{\infty}$ defines the resultant, normalized by the free stream, incident flow $V^{*}=\left(V_{X}^{*}, V_{Y}^{*}\right)$ (with respect to the middle point of the blade) at each angular position $\beta$, with components

$$
\begin{aligned}
& V_{X}^{*}=1-2 k l_{2}^{*} \sin (\beta) \beta_{A} \sin \left(2 k t^{*}\right) \\
& V_{Y}^{*}=-2 k l_{2}^{*} \cos (\beta) \beta_{A} \sin \left(2 k t^{*}\right),
\end{aligned}
$$

and effective incidence angle $\alpha$, defined as

$$
\alpha(t)=\arctan \left(\frac{V_{Y}^{*}}{V_{X}^{*}}\right)-\beta .
$$

These parameters are shown in Fig. 1 for a fixed angular position of the oar $\beta$.

\section{Basic Equations and Numerical Method}

Since the blade in its stroke performs pitching oscillations with high amplitude, a noninertial rotating coordinate system fixed to the blade is introduced. In this 
rotating frame, the vector form of Navier-Stokes (NS) equations is :

$$
\begin{aligned}
& \frac{\partial \vec{q}}{\partial t}+\frac{1}{2} \vec{\nabla}(\vec{q} \cdot \vec{q})-\vec{q} \times(\vec{\nabla} \times \vec{q})+\frac{\partial \vec{\Omega}}{\partial t} \times \vec{r} \\
& +2 \vec{\Omega} \times \vec{q}+\vec{\Omega} \times(\vec{\Omega} \times \vec{r})=-\vec{\nabla} p+\frac{1}{R e} \nabla^{2} \vec{q} .
\end{aligned}
$$

The velocity vector $\vec{q}$ is relative to the rotating coordinate system fixed to the tip of the blade located by the vector $\vec{r}$ with respect to the origin of inertial frame of reference the pivot point location. $\Omega$ is the angular velocity of the blade during a stroke. Used notations are :

$\vec{q}$-dimensionless velocity;

$U_{\infty}$-free stream velocity;

$c$-chord length of blade;

$R e$-Reynolds number $U_{\infty} c / \nu$;

$p$-pressure.

Computational domain is defined in polar coordinates $(r, \theta)$. In this domain, the NS Eqs. are rewritten in terms of the vorticity $\omega$, with the stream function $\Psi$ defined by

$$
r^{2} \frac{\partial^{2} \Psi}{\partial r^{2}}+r \frac{\partial \Psi}{\partial r}+\frac{\partial^{2} \Psi}{\partial \theta^{2}}=-\boldsymbol{J} r \omega
$$

where $\boldsymbol{J}\left(\frac{x, y}{r, \theta}\right)$ is the Jacobian. The coordinates $x, y$ are defined in the moving frame of reference fixed with the blade.

In order to generate the computational domain, the classical Joukowski transformation is used. On the surface, the constraint of no slip is applied to define the surface vorticity. The flow at the far downstream boundary is governed with first order differential relations obtained from the tangential NS Eqs. by dropping the viscous term. For the upstream condition, free stream velocity is assumed. Both vorticity and stream function Eqs. are subjected to periodicity in $\theta$-direction. The difference between the formulation used here and $\mathrm{in}^{6)}$ is that the location of the upstream and the downstream boundary is changing during the stroke of the blade with respect to the resultant oncoming stream.

The vorticity equation is solved by an implicit factored method. The truncation error is $O\left[(\Delta r)^{2}+(\Delta \theta)^{4}\right.$ $\left.+(\Delta t)^{2}\right]$, where $\Delta r, \Delta \theta$ and $\Delta t$ are space and time increments respectively. The Fourier transform method is used to solve the stream function equation by direct approach. The vorticity and stream function equations are solved sequentially. The step is repeated until the convergence of surface vorticity is reached.

The surface pressure distribution is obtained by integrating the tangential component of NS Eqs. For further details one should refer to the papers ${ }^{6,7)}$, since similar formulation was used to investigate the unsteady flow around a pitching 2D hydrofoil.

Nondimensional coefficients are defined in global inertial frame fixed with the boat (subscript I) as :

$$
C_{X}=\frac{F_{X}}{0.5 \rho U_{\infty}^{2} C}, C_{Y}=\frac{F_{Y}}{0.5 \rho U_{\infty}^{2} C}, C_{M}=\frac{M}{0.5 \rho U_{\infty}^{2} C^{2}},
$$

where, $F_{X}$ and $F_{Y}$ are the forces acting on the blade in $X$ and $Y$ direction (Fig. 1) and $M$ is the moment. Components of these forces with respect to the pressure and skin friction are :

$$
\begin{aligned}
& C_{X P}=\frac{2}{L} \int_{0}^{2 \pi} p \frac{\partial y_{I}}{\partial \theta} d \theta, C_{X F}=\frac{2}{R e} \int_{0}^{2 \pi} \omega_{I} \frac{\partial x_{I}}{\partial \theta} d \theta, \\
& C_{Y P}=-\frac{2}{L} \int_{0}^{2 \pi} p \frac{\partial x_{I}}{\partial \theta} d \theta, C_{Y F}=-\frac{2}{R e} \int_{0}^{2 \pi} \omega_{I} \frac{\partial y_{I}}{\partial \theta} d \theta, \\
& C_{M P}=\frac{2}{L^{2}} \int_{0}^{2 \pi} p\left(x_{I} \frac{\partial x_{I}}{\partial \theta}+y_{I} \frac{\partial y_{I}}{\partial \theta}\right) d \theta, \\
& C_{M F}=\frac{2}{R e L} \int_{0}^{2 \pi} \omega_{I}\left(-y_{I} \frac{\partial x_{I}}{\partial \theta}+x_{I} \frac{\partial y_{I}}{\partial \theta}\right) d \theta .
\end{aligned}
$$

The coefficients of the normal force and the force acting along the chord length are defined as:

$$
\begin{aligned}
& C_{N}=-C_{X} \sin (\beta)-C_{Y} \cos (\beta), \\
& C_{C}=-C_{X} \cos (\beta)+C_{Y} \sin (\beta) .
\end{aligned}
$$

The hydrodynamical propulsive efficiency of the motion of the boat with respect to the energy used to produce blade stroke is defined as:

$\eta=\frac{\text { work done by propulsive force }}{\text { energy lost to create this force }}$

with respect to the pivot point location and in the global inertial coordinate system. During the present study, it is assumed that the boat is moving steadily with speed $V$. It defines free oncoming stream $U_{\infty}=-V$. If the path of the boat for the time $t$ is described with the relation $S_{X}=-U_{\infty} t$ with angular oar position $\beta(t)$ (Fig. 1) defined with Eq. ( 2 ), the work done by propulsive force $W_{T}$ and its coefficient $C_{P T}$ should be defined as

$$
\begin{aligned}
& W_{T}=\frac{1}{\tau} \int_{0}^{\tau} F_{X} \frac{d S_{X}}{d t} d t=-\frac{U_{\infty}}{\tau} \int_{0}^{\tau} F_{X} d t, \\
& C_{W T}=-\frac{2}{\rho U_{\infty} C} \frac{1}{\tau} \int_{0}^{\tau} F_{X} d t=-\frac{U_{\infty}}{\tau} \int_{0}^{\tau} C_{X} d t,
\end{aligned}
$$

where $\tau$ is the time for a stroke.

The work done by torque force $P_{Q}$ and its coefficient $C_{P Q}$ are defined as

$$
\begin{aligned}
& P_{Q}=\frac{1}{\tau} \int_{0}^{\tau}\left(M \frac{d \beta}{d t}\right) d t=\frac{1}{\tau} \int_{0}^{\tau} M \Omega d t, \\
& C_{P Q}=\frac{2}{\rho U_{\infty}^{2} C^{2}} \frac{1}{\tau} \int_{0}^{\tau} C_{M} \Omega d t .
\end{aligned}
$$

Propulsive efficiency is defined as the ratio:

$$
\begin{aligned}
\eta & =\frac{W_{T}}{P_{Q}}=\frac{-\frac{U_{\infty}}{\tau} \int_{0}^{\tau} F_{X} d t}{\frac{1}{\tau} \int_{0}^{\tau} M \Omega d t} \\
& =\frac{1}{2 \beta_{A} k} \frac{\int_{0}^{\tau} C_{X} d t^{*}}{\int_{0}^{\tau} C_{M} \sin \left(2 k t^{*}\right) d t^{*}},
\end{aligned}
$$

where $t^{*}$ is normalized time, at catch position $t^{*}=0$ and at finish $t^{*}=\tau$ (period for a stroke). In order to estimate the variations of the efficiency per blade stroke an efficiency coefficient $C_{\eta}$ is defined as :

$$
C_{\eta}=C_{X} V / C_{N} v \text {. }
$$

\section{Computational Parameters and Grid Resolu- tion}

The parameters used in the present calculations are :

- Grid resolution : 
number of points in $\theta$-direction-64 (66 with overlapping stations)

number of points in $r$-direction- 42 ;

- Time increment $\Delta t^{*} \in[0.04421,0.01382]$ (varied) ;

- Computational domain-with diameter 15 chord lengths $c$;

- Average number of iteration for solving the vorticity and stream function Eqs. per a time step $N_{\text {iter. }}$ $=50$.

Fig. 3 shows the streamlines for finer grid system (130 $\times 84)$. The streamlines, which are presented in the rotating frame, are the flow near the catch and the finish. These results correspond to the results in Fig. 4a) and $\mathrm{j}$ ) for the grid resolution $(66 \times 42)$. The time increment for both cases was the same. Near the catch, the flow is qualitatively the same for both cases. In case of the higher grid resolution, the structures are better resolved. Some differences in the intensity of the structures is also observed. More precise analysis shows some lag in time. It seems that in case of fine grid the flow is developing a little faster. Analysing the flow pattern near the finish, similar conclusions may be made. Although, the differences into the flow pattern seems to be a little stronger, the flow phenomena are quite comparable. Here again some lag in time is observed according to the convection of main vortex under the blade and the separation near the root of the blade.

Comparison of the force coefficients shows that conclusions made in $^{6)}$ are valid here. Observed oscillations in obtained forces as consequence of decreased speed of convergence in case of coarse grid, follow the mean value (in $10 \%$ range) obtained using twice higher resolution.

\section{Results and Discussions}

\section{1 General Flow Evolution}

The flow around the blade of rowing oar will be strongly affected by the particular choice of the main governing parameters described in the previous section. Before we try to analyse the influence of these parameters on the unsteady flow pattern, induced forces and blade performance, a general picture of the unsteady flow elolution and the common features of the flow development should be explained. For this purpose, the

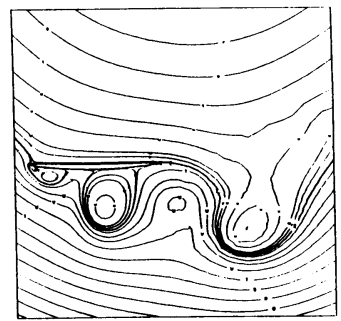

a) $\beta=43[\mathrm{deg}]$

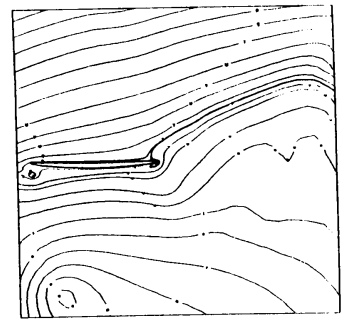

b) $\beta=108[\mathrm{deg}]$
Fig. 3 Streamlines in Rotating Frame. Grid Resolution $(130 \times 84)-$ Standard Case. study for the case, mostly equivalent to the usual cases observed in the rowing practice, is presented.

A simple symmetric cambered profile is selected, with $5 \%$ maximum camber value. Such a shape is quite realistic and is used in practice for some oars. The value of the other governing parameters as the angular velocity of the blade (reduced frequency $k$-Eq. 3 ), the outboard oar length (pivot point location $r$ ), the mean incidence $\beta_{M}$ and the amplitude of pitching $\beta_{A}$, were chosen in order to have a realistic blade motion. In our pilot simulation, these parameters have the values $k=$ 0.081 , normalized pivot length $r^{*}=5.0$ chord lengths, $\beta_{M}$ $=90[\mathrm{deg}], \beta_{A}=90[\mathrm{deg}]$. This case will be referred as a "standard case" here after. In Fig. 6 , the simulated resultant incident flow to the blade ( $V^{*}$, Fig. 1-Eqs. (5), (6)) is compared with the image-processed fullscale data ${ }^{3)}$, which will be discussed in the next section.

Fig. 4 shows the unsteady flow for this standard case, using the velocity vectors and the streamlines defined in the noninertial frame of reference (Oxy Fig. 1) fixed to the tip of the blade, for different angular positions of the oar. In case of the velocity vectors, the blade shows the actual angular position of the oar. The pivot point is located into the direction of the bottom side when the tip of the blade is facing to the top. In the cases when the results are visualized by the streamlines, the pivot point is always to the right and the tip is close to the left side. The blade is moving in clockwise direction (see Fig. 1).

At the initial stage, strong flow separation occurs at the blade tip and vortices are generated successively. At about the catch $(\beta=45[\mathrm{deg}])$, the separated vortical flow still reattaches to the blade. However, the separation vortex, which is generated at the first, is washed downstream (Fig. 4a)) and affects the wake pattern. The intensity of the separation grows further and gradually, the separation region covers the pressure side of the blade (Fig. 4b)). The primary and secondary vortical structures under the blade are merging in one formation. With the continuation of this process, and with respect to the intensity of separation vortex behind the blade root, a trailing edge dynamic stall may occur (Fig. 4c)). The separation vortex slowly convects downstream leaving space for a new tip vortex bubble. At the same time it affects the flow at the root edge enforcing the reverse flow around the root of the blade and trailing edge separation (Fig. $4 \mathrm{~d}$ ), e)). The resultant merged main vortex is following the blade motion and moves further down to the blade root preventing larger separation around the root edge. The process of slow diffusion is also observed (Fig. 4f)). Soon after this moment, the effective incidence starts to change its direction in a very drastic way from the tip to the root of the blade Fig. 4f), g) and h)). This process is attended by the weakening of tip separation when the root separation is influenced greatly by the main vortex structure. With respect to the reduced frequency, the 


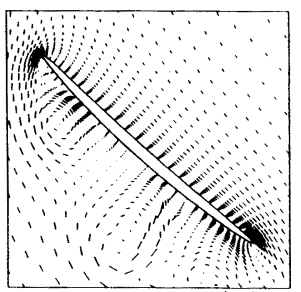

a) $\beta=43[$ deg $]$

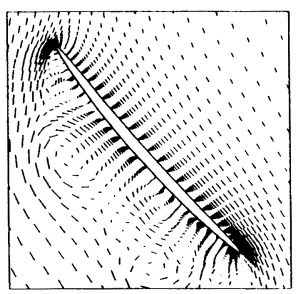

b) $\beta=49[\mathrm{deg}$

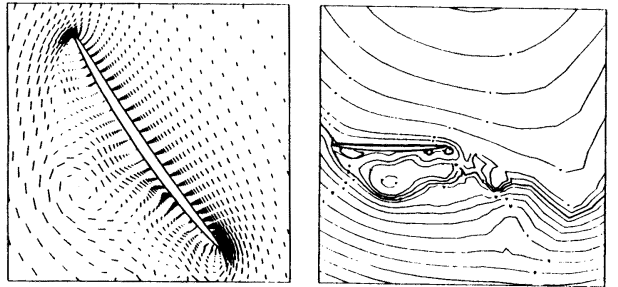

c) $\beta=56[$ deg $]$
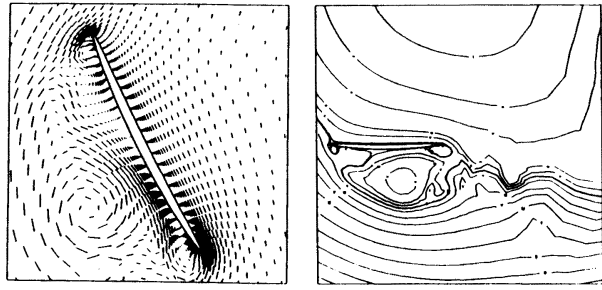

d) $\beta=63[\mathrm{deg}]$
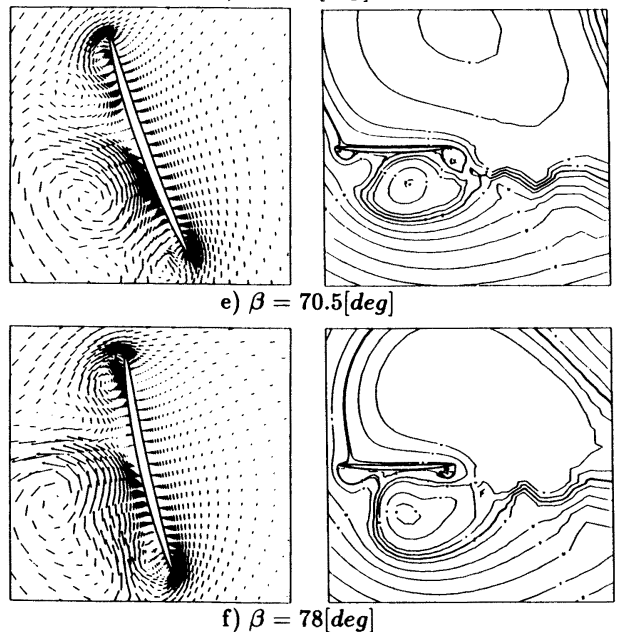

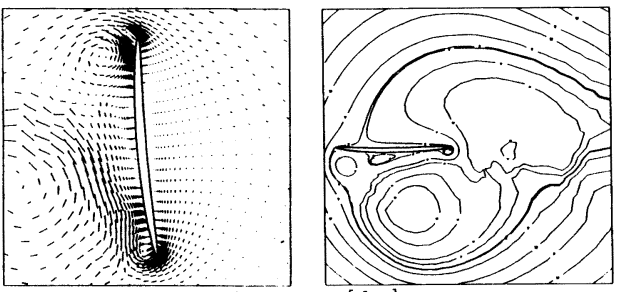

g) $\beta=85.5[\mathrm{deg}]$

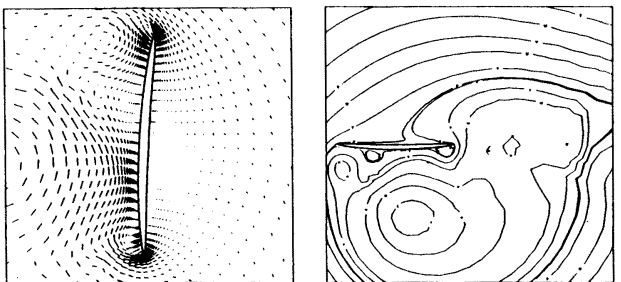

h) $\beta=93[\mathrm{deg}]$
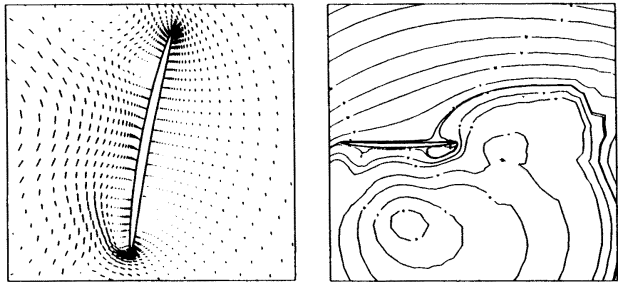

i) $\beta=101[\mathrm{deg}]$

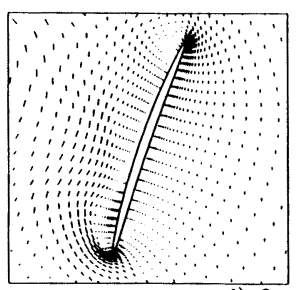

$$
\text { j) } \beta=108[\text { deg }]
$$
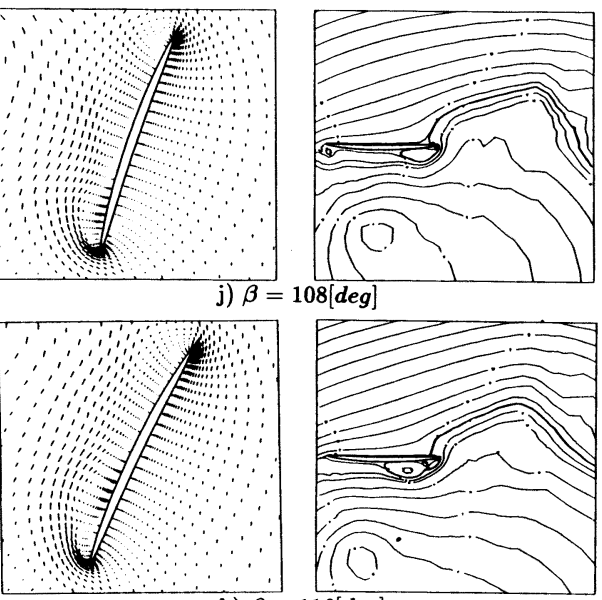

k) $\beta=116[\mathrm{deg}]$
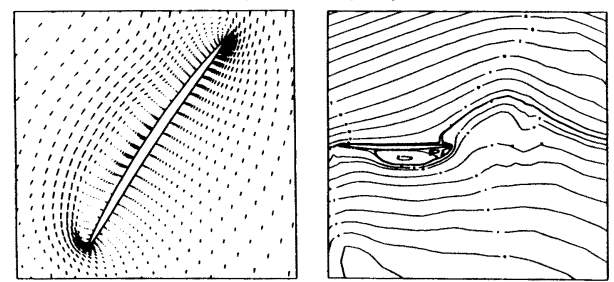

l) $\beta=123[\mathrm{deg}]$

Fig. 4 Velocity Vectors and Streamlines in Rotating Frame. Standard Case. 
length of oar, etc., the vortices shedded into the wake and convected under the blade root may influence the oncoming flow and root separation as well. The flow develops further and by the impinge of the resultant flow on the fluid around the blade root, the main vortex structure detaches from the blade and is convected slowly back in the direction of the blade tip (Fig. 4i)) The influence of shedded vortices is weakening. The root separation is growing. This tendency continues in Fig. $4 \mathrm{j}), \mathrm{k}$ and $\mathrm{l}))$. At the finish $(\beta=120[\mathrm{deg}])$ no tip separation is observed. The separation is prevented by the main vortex, convected gradually under the tip of the blade, which improves the reattachment of the root separation.

The unsteady effects may have strong influence on the flow behavior. To assist this concept, the flow around the blade with the same shape and fixed incidence of 60 [deg] was studied numerically. The chord Reynolds number during this study is 3000 . The developed flow for this case is oscillating. The typical streamlines, shown in Fig. 5, correspond to normalized time $t^{*}=14$ (time, at which the blade motion is stopped, is taken as $t^{*}=0$ ). The vortices are shedding into the wake alternatively. This case will be referred hereafter as 'steady state', which means the oar is fixed to some angle. The corresponding unsteady streamlines are shown in Fig. $4 \mathrm{~g}$ ). Even brief look at these results shows that the unsteady flow is phenomenologically different from the flow of steady state case.

The results for the normal force coefficient $C_{N}$ and the efficiency coefficient $C_{\eta}$ for the present "standard case" are presented in the next sections of the discussion as a comparison with the cases with different governing parameters.

\subsection{Angular Velocity}

Here, it is investigated how the flow will behave in case of different reduced frequency $k$, namely, the different rowing speed compared with the boat speed. During the study, three different cases are compared. The first one, with reduced frequency $k=0.081$ (stan-

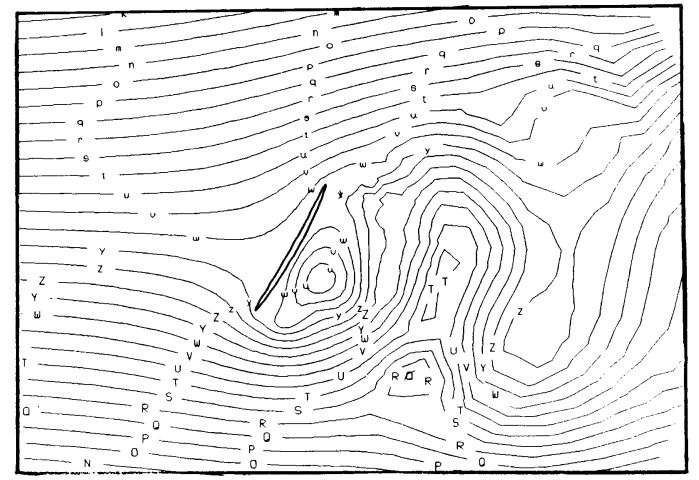

Fig. 5 "Steady State" Streamlines around the Blade. Angle of Attack $=60[\mathrm{deg}]$. dard case), the second, with smaller reduced frequency $k=0.071$ (slow rowing compared with the boat speed) and the third, with much larger reduced frequency $k=$ 0.1 (fast rowing). Other parameters will be the same for the three compared cases. Normalized resultant incident flow during the blade stroke for these cases is shown in Fig. 6. Analysing the resultant incidence velocities (Eqs. (5), (6)) reflecting in effective angles of attack (Eq. ( 7$)$ ), it have to be noted that the differences are remarkable.

Fig. 7 shows the flow at three angular positions of the oar, one near the catch angle, second, approximately in the middle position, and third-near the finish. The flow is presented by means of velocity vectors and streamlines in the noninertial frame fixed to the blade. These results concern the case of slow rowing. The flow for the fast rowing is presented in Fig. 8 at approximately same angular positions. These results may be compared with the results for the standard case presented in Fig. $4 \mathrm{~b}), \mathrm{g}$ ) and k) respectively.

With the increase of the rowing speed, the flow separation near the blade tip and root is larger and the flow develops faster (Fig. 7a), Fig. 4b) and Fig. 8a)). In the case of slow rowing, the flow still reattaches to the blade, when at high reduced frequency the flow is completely separated and drastically different flow pattern is observed. This tendency develops further. In the slow case, the primary separation vortex is followed by the secondary one and this vortex pair sticks in the region under the blade. In the case of fast rowing, the frequency of blade tip vortex formation, their intensity, and the speed of their convection is much higher. Some of these vortices formed at the initial stage of flow separation are shedding into the wake and are convected below the root of the blade (see Fig. 8b)).

Near the finish for the slow rowing case, the vortex pair is merged in one structure which accelerates the flow over the back side of the blade (Fig. 7c)). For the standard case, this merged vortical structure is convect-

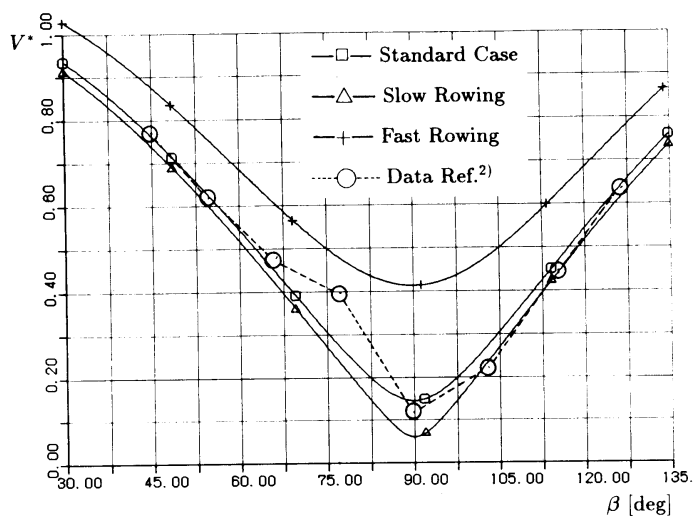

Fig. 6 Resultant Incident Velocity per Stroke. "Speed of Rowing" Effects. 

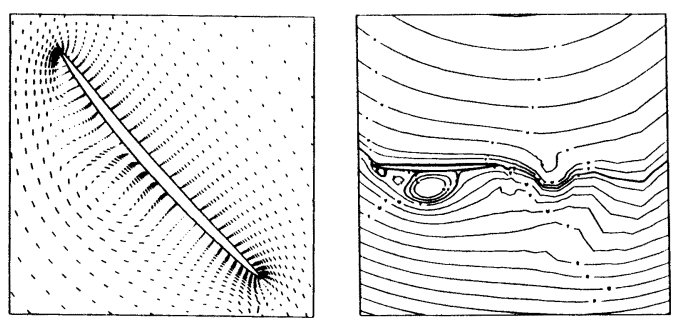

a) $\beta=49[\mathrm{deg}]$
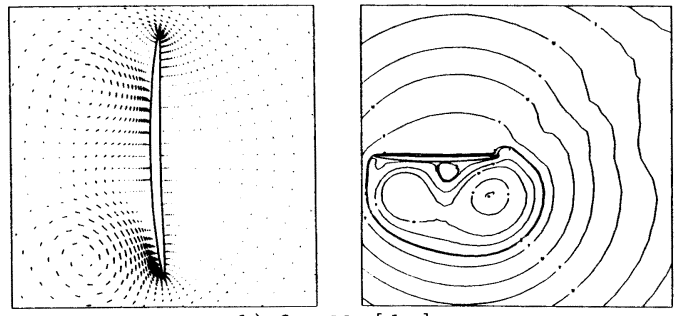

b) $\beta=89.5[\mathrm{deg}]$
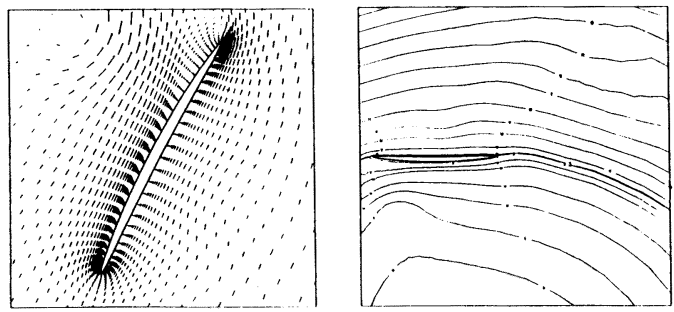

c) $\beta=117[\mathrm{deg}]$

Fig. 7 Velocity Vectors and Streamlines in Rotating Frame. Slow Rowing.

ed back to the blade tip, when the separation around the blade root is developing (Fig. 4k). In the case of fast blade rowing, the separation bubbles, which are formed near the blade tip and are convected to the blade root at the previous stage, are not detached and are not washed out. This flow becomes the core of the separation at the root edge of the blade (Fig. 8c)). The vortex shedded at the previous stage accelerates the oncoming flow in this region.

In Figs. 9 and 10, a comparison is made of the coefficients of the normal force $C_{N}$ and efficiency coefficients $C_{\eta}$ for the studied cases. The magnitude of the force increases with the increasing of rowing speed. The coefficient for the standard case is in general between the values for the slow and fast rowing. The coefficient at the finish is larger than near the catch position with the exception of the case slow rowing. The clean finish with almost zero effective incidence in the case of slow rowing leads to relatively small force induction but at higher efficiency. The slowest rowing is the most efficient when the fastest rowing is the most inefficient one. The last section of the discussions treats the efficiency in more details. For faster boat speed we need faster rowing, which consumes more power to be produced.
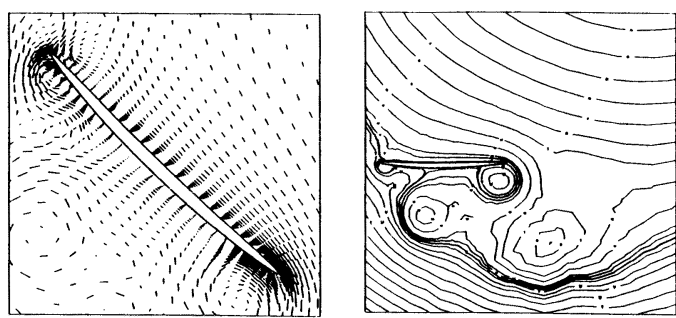

a) $\beta=45[\mathrm{deg}]$
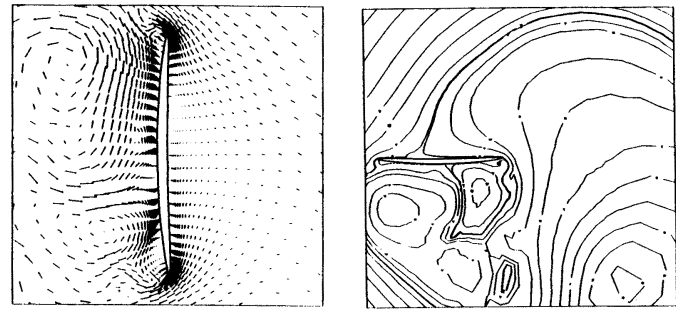

b) $\beta=89.5[\mathrm{deg}]$
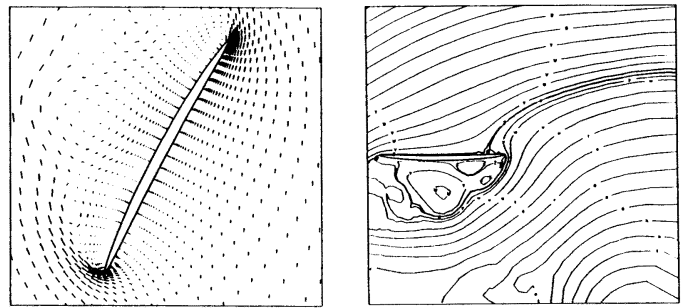

c) $\beta=117[\mathrm{deg}]$

Fig. 8 Velocity Vectors and Streamlines in Rotating Frame. Fast Rowing.

\section{3 Blade Geometry}

In this section it is investigated how the blade geometry will affect the unsteady flow and induced forces. The standard case will be compared with two other cases. The first will have approximately the same geometry profile but with maximum camber of $10 \%$ chord lengthtwice begger than the camber of the blade in the standard case $(5 \%)$. The camber of the blades used in practice varies in the interval [5\%, $7.5 \%]$. The blade geometry in the second case will look a little strange for the people experienced in rowing, as the foil geometry is thicker to the tip than to the blade root. It has average camber of $7.5 \%$. This choice is a result of the preliminary idea that, if we can decrease the separation near the catch position, it may be possible to obtain better efficiency at this stage of the stroke and improve the overall efficiency of rowing. The geometry of the blade for each of the cases is illustrated in Fig. 11 and further will be referred as a) BLADE1 (standard case), b) BLADE2 (big camber) and c) BLADE3. Since the chord length is approximately the same and other parameters have the same values too, the only difference between the studied cases will be the blade geometry moving at the same angular speeds and accelerations. 


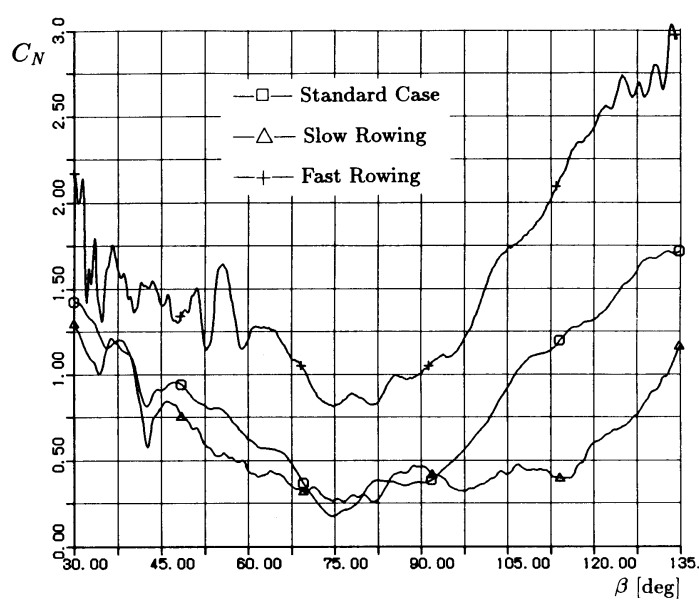

Fig. 9 Normal Force Coefficient $C_{N}$. "Speed of Rowing" Effects.

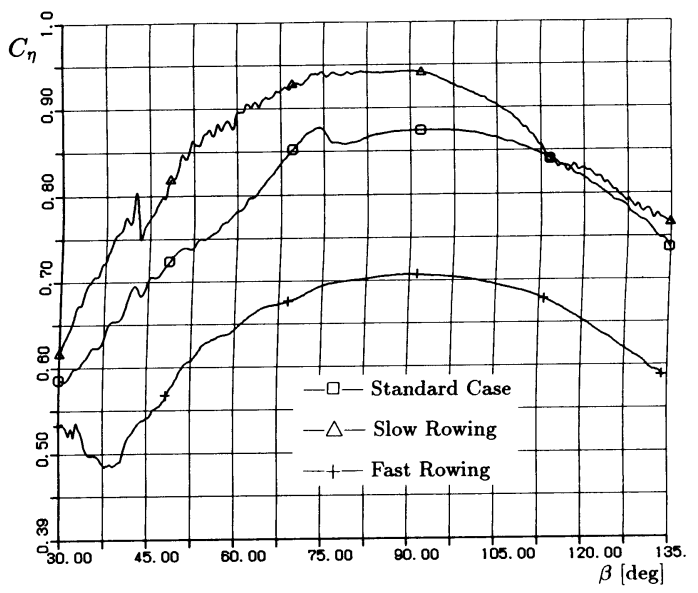

Fig. 10 Efficiency Coefficient $C_{\eta}$. "Speed of Rowing" Effects.
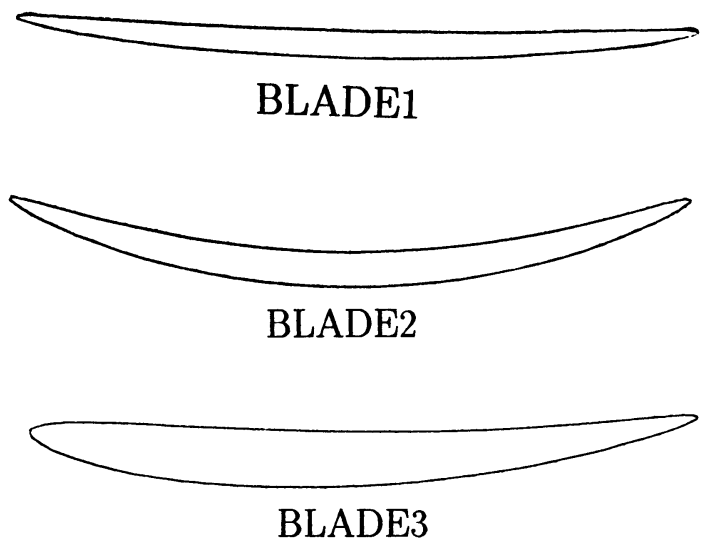

Fig. 11 The Shape of the Blade for the Studied Cases.
Before we go into details about the flow and induced forces, it might be helpful to analyse the experience of the professionals since, at this stage, "blade design is more art than a since". At present moment no strong consensus exists as to the best blade geometry, there is a limited number of expert rules. The one concerning the camber of the blade (blade spoon) is that the blade with larger camber is better at the catch but worse at the finish, when a blade with less camber has more "clean" finish.

Flow analysis will be performed for three selected angular positions of the oar-near the catch, around the middle position and near the finish. Velocity vectors and streamlines in the noninertial frame fixed to the blade are shown for the case of BLADE2 in Fig. 12 and in Fig. 13 for the case of $B L A D E 3$ respectively. These results may be compared with the standard case (BLADE1) in Fig. $4 \mathrm{c}), \mathrm{g})$ and 1). Near the catch position $(\beta=56$ [deg]), in the standard case, the flow is fully separated. Enforced circulation around the root of the blade (so called trailing edge stall) will decrease the normal force. Increasing the camber of the blade stimulates later separation. The separation bubbles are still attached to the foil and have weaker affect on the flow near the blade root and wake. In case of BLADE3 the flow is still able to reattach to the foil after which detaches again facing the primary vortex developed earlier.

Near the middle position $(\beta=85[\mathrm{deg}])$, the flow for all of the cases has much in common and except the larger separation near the blade root in the cases BLADE2 and BLADE3. Such result may be expected as at this moment the differences in the geometry will not affect the flow pattern so much. Observed discrepancies should be explained rather with the different flow history than with the geometry differences.

Near the finish we can expect again stronger geometry dependency. The separation observed at the blade root for the standard case is missing completely in the case of large camber. At the same time strong separation from the middle to the blade tip for this case is observed. This separation will have crucial affect on the force and moment coefficients and we can expect sharp loss of efficiency. For the case BLADE3, the small separation near the blade root is followed by relatively clean blade tip, although the thickening of the boundary layer in this region is remarkable.

These flow patterns have to be connected with the coefficient $C_{N}$ compared in Fig. 14. The smaller flow separation at the catch $(\beta=45[\mathrm{deg}])$ in case of cambered blade facilitates larger forces. This tendency is valid around the middle position as the separation around the edges should be a little stronger in case of more cambered profile. At the finish $(\beta=120[\mathrm{deg}])$, the separation around the blade tip (BLADE2) induces very large force and moment coefficients, followed by abrupt decrease when the separation starts to affect the flow at 


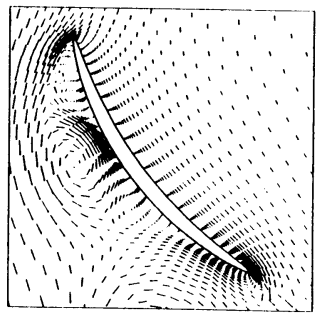

a) $\beta=53[$ deg]

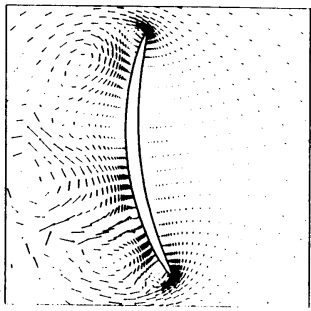

b) $\beta=84[\mathrm{deg}]$
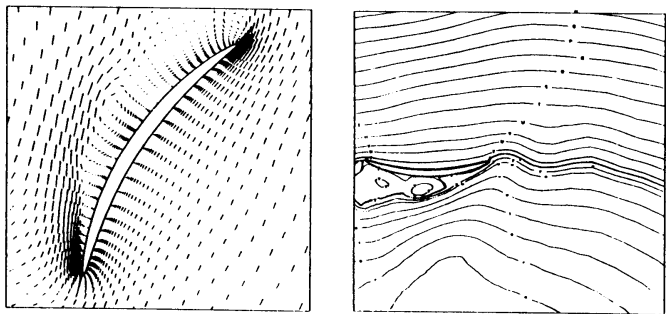

c) $\beta=123[\mathrm{deg}]$

Fig. 12 Velocity Vectors and Streamlines in Rotating Frame. BLADE2 (Big Camber).

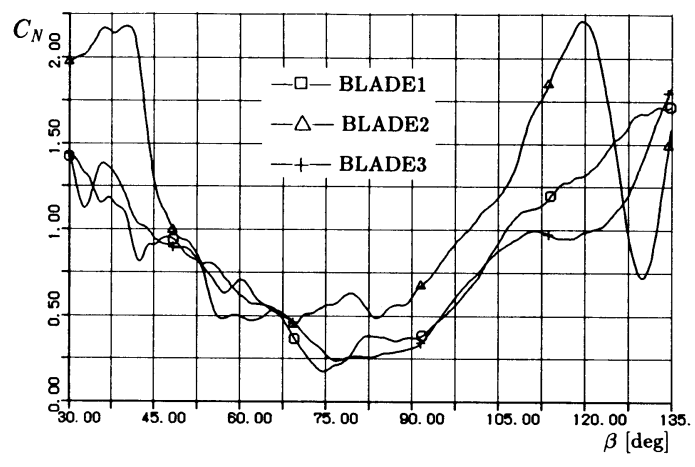

Fig. 14 Normal Force Coefficient $C_{N}$. "Blade Geometry" Effects.

the blade tip (Fig. 12c)) when effective incidence angle at the root of the blade is near zero. Differences between BLADE1 and BLADE3 are less remarkable except the region near the finish. At this moment, due to the clean finish observed in Fig. 13c), larger force coefficients $C_{N}$ are realized.

A comparison of efficiency coefficient $C_{\eta}$ of the studied cases is presented in Fig. 15. Having the results
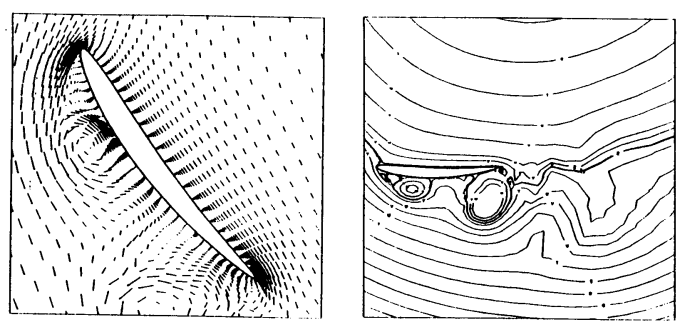

a) $\beta=53[\mathrm{deg}]$
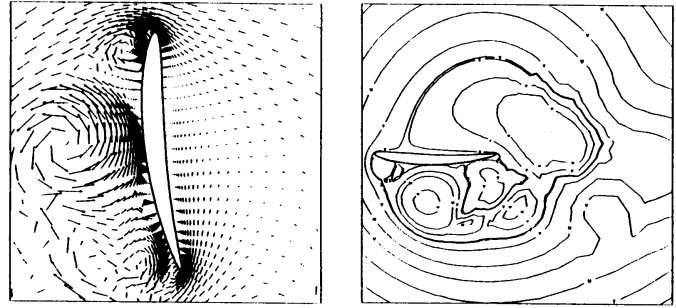

b) $\beta=84[\mathrm{deg}]$
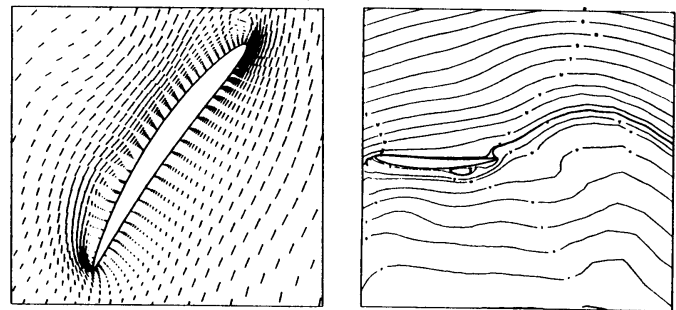

c) $\beta=123[\mathrm{deg}]$

Fig. 13 Velocity Vectors and Streamlines in Rotating Frame. BLADE3.

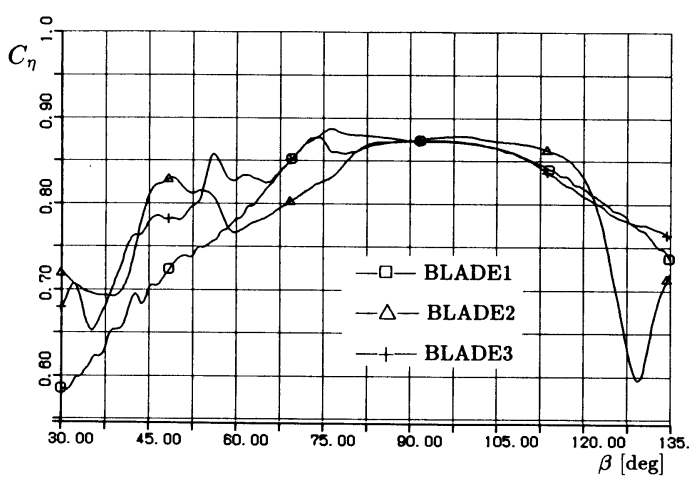

Fig. 15 Efficiency Coefficient $C_{\eta}$. "Blade Geometry" Effects.

about the flow and induced forces, it can be explained that BLADE2 and BLADE3 will have better efficiency near the catch position and a little lower around the middle for the BLADE2. At the finish $(\beta=120[\mathrm{deg}])$ the abrupt loss of efficiency in the case of cambered blade is direct result of the separation at the blade tip. The discussion about the efficiency $\eta$ is presented in 5.5 comparing the influence of all of the parameters. 


\section{4 Acceleration of Blade Motion}

Here, the effect of acceleration of resultant oncoming flow is investigated. Since the velocity at each point of the blade surface is a product of blade angular velocity and a local distance to the pivot point location, the oar outboard length and the blade angular velocity are important parameters governing the blade motion through the fluid. The angular velocity of the blade is selected in the way, that the resultant flow and the effective angle of attack with respect to the middle point of the blade remain the same. As the chord length for the simulation is the same, the resultant incident flow at the tip and root of the blade is different. However, the magnitude of this difference is at most $2 \%$ and we can not expect any strong influence. The main difference is expected different unsteady effects due to the different angular acceleration. Here, the standard case $\left(k=0.081, r^{*}=5.0\right)$ is compared with $10 \%$ shorter and $10 \%$ longer oar outboard length. Corresponding reduced frequences of the stroke are $k=0.091$ and $k=$ 0.073 respectively. Other parameters, as blade geometry, mean incidence and amplitude of pitching, have been kept the same. The angular acceleration of oar is compared in Fig. 16. The velocity field and the stream function in the noninertial moving frame for three
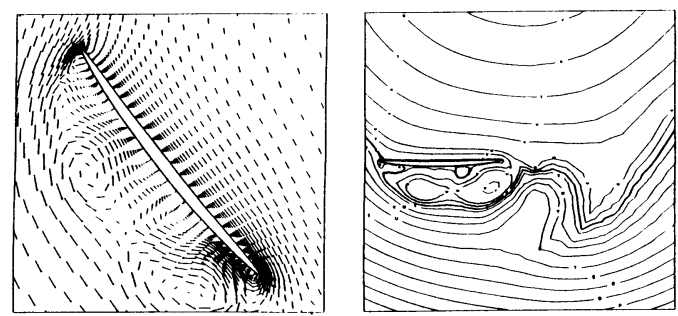

a) $\beta=52.5[\mathrm{deg}]$
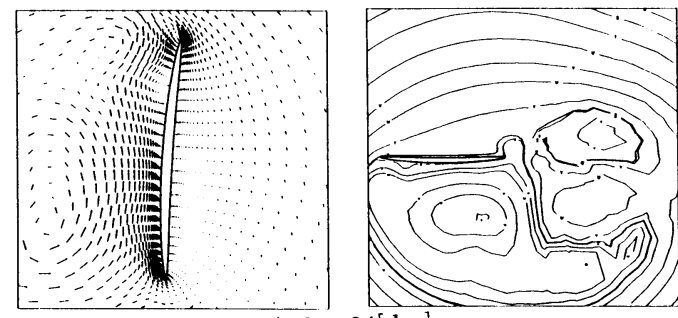

b) $\beta=94[$ deg $]$
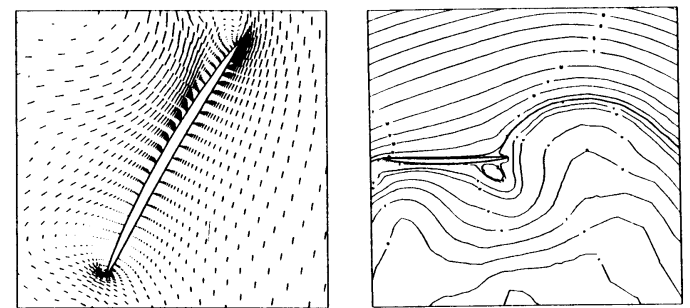

c) $\beta=119[\mathrm{deg}]$

Fig. 17 Velocity Vectors and Streamlines in Rotating Frame. Short Oar. angular positions of the oar are shown in Figs. 17 and 18. The comparison of the coefficients $C_{N}$ and $C_{\eta}$ for the three compared cases is presented in Figs. 19 and 20 . The flow results have to be compared with the flow pattern for the standard case-Fig. $4 \mathrm{~b}$ ), h) and k) respectively.

Near the catch position, the resultant oncoming flow

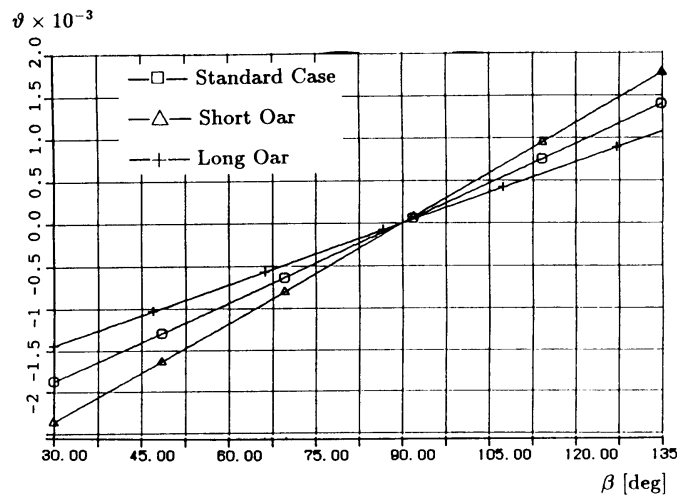

Fig. 16 Angular Acceleration per Stroke. "Outboard Oar Length" Effects.
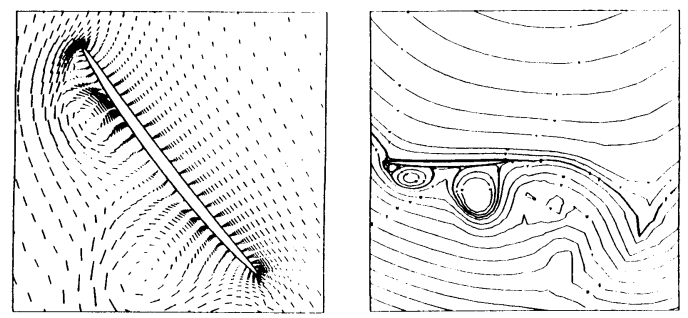

a) $\beta=52.5[\mathrm{deg}]$
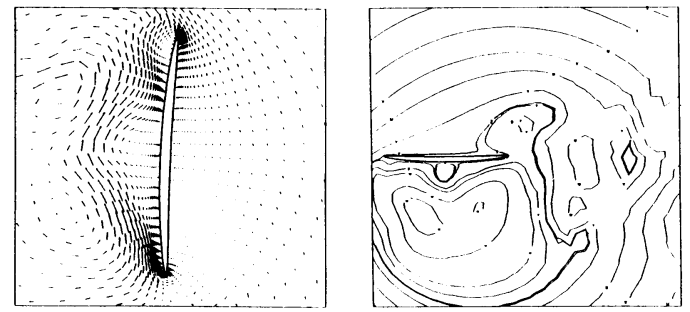

b) $\beta=94[\mathrm{deg}]$

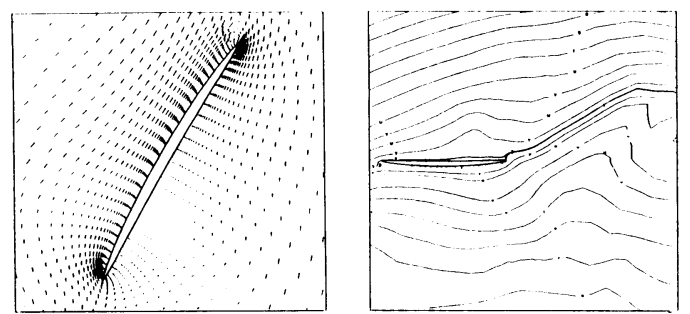

c) $\beta=119[\mathrm{deg}]$

Fig. 18 Velocity Vectors and Streamlines in Rotating Frame. Long Oar. 


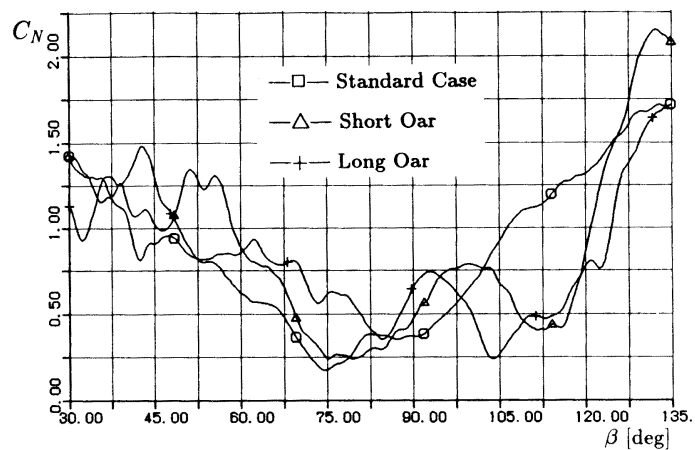

Fig. 19 Normal Force Coefficient $C_{N}$. "Outboard Oar Length" Effects.

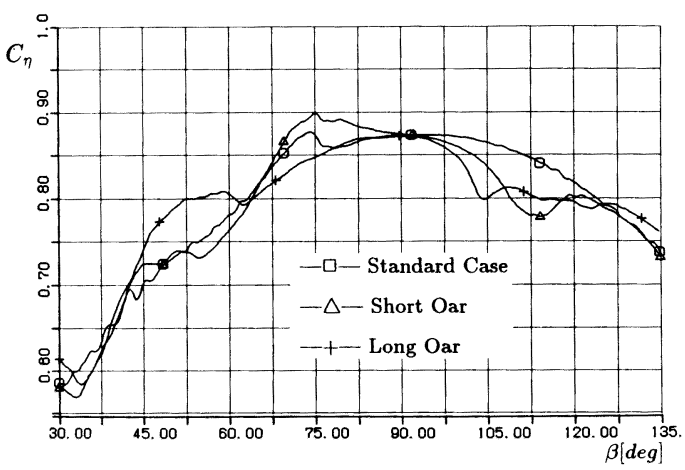

Fig. 20 Efficiency Coefficient $C_{\eta}$. "Outboard Oar Length" Effects.

is decelerating, and this deceleration is smaller for the long oar. So that, the convection of vortices, which are generated at the blade tip, is faster for the longer oar especially behind the blade root (Figs. 17a), 18a), 4b)). These vortices affect the flow around the blade root and the wake remarkably. Further we can expect the influence of the flow prehistory.

Near the middle position $(\beta=90[\mathrm{deg}])$ the difference between the cases is not so big (Figs. 17b), 18b) 4h)).
The effect of acceleration is not dominant at this stage, so that the difference of the flow is mainly caused by the prehistorical flow of the separated vortices.

At the next stage, near the finish, the root of the blade is acting as a leading edge. The angular acceleration of resultant oncoming flow, which is growing at this stage, is larger for the shorter oar. This acceleration may lead to the separation at the root. This effect is observed in Figs. $17 \mathrm{c}), 18 \mathrm{c}$ ) and 41 ). Increasing the length of the oar makes the influence weaker. In the case of long oar, no separation is observed on the back side of the blade and the flow is clean (Fig. 18c)) except the disturbance observed over the face side. This disturbance is produced by the convection of vortices near the catch position (see Fig. 18b)) and leads to the poor force and efficiency (Figs. 19 and 20). In the case of short oar, the force coefficient and efficiency are decreasing near the finish. It is caused by the combined effect of the acceleration and the reverse circulating flow near the root.

Comparison of the coefficients $C_{N}$ for the three compared cases is presented in Fig. 19. Near the catch angle $(\beta=45[\mathrm{deg}])$, the long oar produces the largest force coefficients, because of the clean flow at the root (trailing edge). At the finish, both the short and the long cases have worse performance.

\section{5 Performance}

The hydrodynamical propulsive efficiency of the blade per a stroke (Eq. 21) is estimated neglecting the oar weight, inertial moment, etc. The stroke begins at $\beta$ $=47.4$ [deg] (catch) and ends at $\beta=122.3[\mathrm{deg}]$ (finish). These values are chosen with respect to $^{5}$.

The value of the efficiency and parameters for any particular case studied here, are summarized within the Table 1. To make the analysis easier, the values of the mean thrust coefficient and mean power coefficient, defined by Eqs. (18) and (20) respectively are included.

Comparing the values for all cases, the case No.2, characterized by the slowest blade velocity, appears as the most efficient one. It is a direct consequence of the smallest separation and ineffective dissipation of energy for the vortex production during the stroke. At the same time we have the smallest power consumption

Table 1 Blade Geometry, Governing Parameters and Performance.

\begin{tabular}{||c||c|c|c|c|c|c|c|c||}
\hline \hline $\begin{array}{c}\text { Case } \\
\text { No. }\end{array}$ & $\begin{array}{c}\text { Blade } \\
\text { Geometry }\end{array}$ & $\begin{array}{c}\text { Reduced } \\
\text { Freq. } k\end{array}$ & $\begin{array}{c}\text { Pivoting } \\
\text { Point } r^{*}\end{array}$ & $\begin{array}{c}\text { Mean } \\
\text { Incidence }\end{array}$ & $\begin{array}{c}\text { Amplitude } \\
\beta_{A}\end{array}$ & $\begin{array}{c}\text { Mean Thrust } \\
\text { Coefficient }\end{array}$ & $\begin{array}{c}\text { Mean Power } \\
\text { Coefficient }\end{array}$ & $\begin{array}{c}\text { Efficiency } \\
\eta\end{array}$ \\
\hline \hline 1 & BLADE1 & 0.081 & 5.0 & $90[\mathrm{deg}]$ & $90[\mathrm{deg}]$ & 0.62 & 0.74 & 0.83 \\
\hline 2 & BLADE1 & 0.075 & 5.0 & $90[\mathrm{deg}]$ & $90[\mathrm{deg}]$ & 0.39 & 0.44 & 0.88 \\
\hline 3 & BLADE1 & 0.100 & 5.0 & $90[\mathrm{deg}]$ & $90[\mathrm{deg}]$ & 1.26 & 1.89 & 0.67 \\
\hline 4 & BLADE1 & 0.091 & 4.5 & $90[\mathrm{deg}]$ & $90[\mathrm{deg}]$ & 0.59 & 0.73 & 0.80 \\
\hline 5 & BLADE1 & 0.073 & 5.5 & $90[\mathrm{deg}]$ & $90[\mathrm{deg}]$ & 0.58 & 0.72 & 0.82 \\
\hline 6 & BLADE2 & 0.081 & 5.0 & $90[\mathrm{deg}]$ & $90[\mathrm{deg}]$ & 0.88 & 1.04 & 0.84 \\
\hline 7 & BLADE3 & 0.081 & 5.0 & $90[\mathrm{deg}]$ & $90[\mathrm{deg}]$ & 0.56 & 0.67 & 0.83 \\
\hline \hline
\end{tabular}


with the smallest mean thrust value.

For the case No. 3 (the fastest blade motion) with the worst efficiency, we have the largest separation and vorticity production. The speed of the boat will be the fastest one, but the price of this is a very high power consumption. Going back to Fig. 9 where the efficiency coefficients for the case No. 2 and No. 3 are compared with case No. 1, it is clear that the efficiency of the fast rowing is lower all the way during the stroke and especially near the catch position.

Comparing the case No. 1 (standard), No. 4 and No. 5 (outboard oar length) we can see that the shortest oar is inefficient. Efficiency is increasing to some limits (near standard case) and is going down again with the further increase of the length of the oar. Referring Fig. 20, we have to keep in mind that the angular velocity of the blade (and acceleration) is different for these cases in order to compensate the differences in the oar length. If we kept the angular velocity of the blade the same, the shortest oar will be the most efficient one according to the discussion for the blade velocity effects. The values of the mean thrust and mean power are comparable with the standard case.

The comparison of the cases with different blade geometry (No.1, No. 6 and No. 7) needs more studied cases and precise analysis as the efficiency for these cases is comparable. The efficiency is complex function of many parameters and it is hard to predict their combined affect on blade performance. From this point of view, flow phenomena understanding seems to be important and valuable.

\section{Conclusions}

The unsteady viscous flow around 2D blade of rowing boat is studied numerically. To investigate the common flow features and to reveal qualitatively the sensitivity of the flow, induced forces and efficiency, a parametric study is performed. The following conclusions can be made.

- Flow analysis proves that the simulated flow has highly unsteady nature. The main factors governing the flow development are connected with the oncoming flow acceleration, the sharp changes in the effective incidence and the strong influence of flow history.
- The dependence of the flow and induced forces to the shape of blade shows promising ways to increase the efficiency. A blade with higher curvature is efficient at the catch, but inefficient at the finish.

- The case of slow blade motion (slow rowing) is found as the most efficient. High efficiency is coupled with low power consumption and limited propulsive abilities.

- The strong influence of flow acceleration on the unsteady flow evolution, analized in connection with the oar length, points that the boat acceleration might have an important affect on the performance. Additional study of this problem is desirable.

- Performed grid resolution analysis shows that the accuracy of the flow simulated with the present grid system is qualitatively comparable with the finer grid case. Such limited grid resolution can be used for the pilot investigations.

\section{References}

1) Ikehata, M., Kajitani, H. "On the Design of Shell Eight Hull for Rome Olympic Games”, SENPAKU, Vol. 34, No. 1, 1961, pp. 35-54. (In Japanese)

2) Hayashi, I., Fukuda, J. and Yamada, H., "The Influence of Shapes and Sizes of Oar on the Driving Force of Shell-Four Boat Using a Dynamic Numerical Model", J. of Human Science, Vol. 3, No. 2, 1990, pp. 17-32. (in Japanese)

3) Amo, K., Fujikami, Y., "Study on the Hydrodynamic Forces Generated by the Oar of Shell Eight Rowing Boat", Graduation Thesis of Hiroshima University, 1991. (in Japanese)

4) Mori, K., Doi, Y., Nakashima, H., "Hydrodynamic Analysis of the Forces Generated by the Oar of Shell Eight Rowing Boat", J. Soc. Naval Arch. of Japan, Vol. 170, Mar. 1992, pp. 103-110. (in Japanese)

5) Suzuki, K., "Technological Approach to Rowings of Shell Eight Boat", J. Kansai Soc. N. A. of Japan, No.218, Sep. 1992, pp. 121-132. (in Japanese)

6) Videv, T. A., Doi, Y., "Numerical Study of the Flow and Thrust Produced by a Pitching 2D Hydrofoil", J. Soc. Naval Archh. of Japan, Vol. 172, Nov. 1992, pp. 165-174.

7) Videv, T. A. and Doi, Y., "Numerical Study on Unsteady Flow Around Oscillating Hydrofoil", Proceedings of the 5th Conference on CFD, Tokyo, 1991 pp. 255-258. 